\title{
Spontaneous Intraperitoneal haemorrhage in Dengue fever
}

\author{
Zakeer $M S^{1}$, Ghetheeswaran $S^{1}$, Udayakumaran $S^{1}$ \\ ${ }^{1}$ Teaching Hospital Jaffna. \\ Corresponded Author: Zakeer, M.S. \\ Email: zakeersamoon@gmail.com
}

https://orcid.org/0000-0003-3747-5694

\begin{abstract}
Dengue Haemorrhagic Fever (DHF) can be very diverse in presentation ranging from asymptomatic petechial skin haemorrhages to lifethreatening intracranial,pulmonary, gastrointestinal and genitourinary haemorrhages. There have been several case reports of dengue haemorrhagic fever with intraperitoneal haemorrage secondary to splenic rupture,but only few cases with normal spleen and normal clotting profile, here we report a case in pregnant women with dengue haemorrhagic fever complicated by spontaneous intraperitoneal haemorrhage.
\end{abstract}

\section{Introduction}

Dengue fever is caused by four distinct dengue virus types, 1, 2, 3, and 4 belonging to the genus flavivirus of family togaviridae. The impact of dengue illness on the healthcare system of Srilanka is enormous. (1) Dengue fever has an incubation period of 4-7(range3-14) days, is clinically characterized by fever, retro-or bital pain, myalgia, arthralgia and generalized erythema. Bleeding manifestations like petechial rashes, gumbleeding, subconjunctival hemorrhage and severe bleeding manifestations like epistaxis, hematemesis, hematuria and hemoperitoneum are also observed.

There are few case reports of dengue fever with hemoperitoneum secondary to spontaneous rupture of spleen. (3) We report a case of serologically confirmed dengue fever with hemoperitoneum with normal spleen and normal coagulation profile. And as per the available literature search this is the second such case reported.

\section{Case Report}

A 40 year old pregnant women from Pannalai area in Jaffna with POA of eleven weeks was transferred from the local hospital to Teaching hospital Jaffna with complaints of fever of five days with myalgia, headaches, dizziness, and abdominal pain with rapid distension. She did not have any co-morbid illnesses, no history of any drug abuse and did not have any history of trauma. She did not have any external bleeding manifestations. On examination she was afebrile, pale, anicteric ,her pulse was 135 beats/min with a blood pressure of 130/80. Abdominal examination revealed diffuse tenderness. Other systemic examination was unremarkable. The full blood count at local hospital showed a hemoglobin of $7.6 \mathrm{~g} / \mathrm{dl}$ with platelet of $106 \times 10^{9}$, her baseline haemoglobin on day of admission was $12 \mathrm{~g} / \mathrm{dl}$. A working diagnosis of dengue hemorrhagic fever was made, We took bloods for all routine investigation, clotting profile did an urgent crossmatching of blood and started her on IV fluids. USS of abdomen which showed a large intraperitoneal haematoma of $17 \times 11.7$ $\mathrm{cm}$,bilateral small pleural effusion, gallbladder edema consistent with fluid leakage and the spleen was normal. The clotting profile was normal and the blood picture showed features of anemia secondary to acute blood loss with no features of DIC. The dengue antibody IgM and IgG were positive. She was stated on IV Tranexamic acid and required a total of five pints of blood and four units of cryoprecipitate. An obstetric referral was done and her fetus was viable. Her vital parameters improved and abdominal tenderness and girth reduced gradually, she was feeling better over the course of her stay and on the tenth day after her admission we repeated the USS abdomen which showed reduction of the size of haematoma. Her blood counts normalised and the recovery was unevent full.

\section{Discussion}

The clinical spectrum of dengue fever can range from asymptomatic infection to life threatening dengue hemorrhagic fever (DHF) and dengue shock syndrome (DSS). There are various theories of the pathogenesis of DHF/DSS, such as increase in vascular permeability, perivascular edema, vascular endothelial injury and parenchymal necrosis with splenic hyperplasia. In this patient being pregnant has also implications in dengue as studies have shown that clnical aspect of dengue is 
different from that in nonpregnant state. In Brazil two studies showed that pregnant women with dengue were three times more likely to develop severe dengue or die due to this than non pregnant women. (2) A combination of (a) increased prothrombin time (b)hemoconcentration (c) platelet count of less than 50,000cells/mm3 and (d) elevated alanine transaminase (ALT) is known to be predictive for spontaneous bleeding manifestations. (3) Hemoperitoneum in dengue fever though rare can be life threatening if not recognized early. In our patient an intraabdominal bleeding was suspected as patient had a rapidly dropping hemoglobin with tachycardia and increasing girth of abdomen, which prompt us to do an ultrasound scan of abdomen which confirmed it. But sometimes it is difficult to differentiate between hemoperitoneum and transudative ascites, so regular monitoring of vital signs, regular full blood count are important in a patient with dengue fever for early detection of internal bleeding and associated complications.

\section{References}

1. Guidelines on management of Dengue fever in adults, Ministry of Heallth, Srilanka.National guidelines revised and expanded edition November 2012.

2. Enny S Paixao, Katie Harron,et al. Dengue in pregnancy and maternal mortality: a cohort analysis using routine data July 2018 Nature https : // doi.org / 10.10 38/ s41598 - 018 $28387-w$

3. Chandrashekar NT, Krishnappa R, Reddy CS, Narayan A. Hemope -ritoneum in dengue fever with normal coagulation profile. J Global Infect Dis 2013;5:29 - 30 